\title{
GLB1-related disorders: GM1 gangliosidosis and Morquio B disease
}

\author{
Sung Yoon Cho ${ }^{(1)}$ and Dong-Kyu Jin*(i) \\ Department of Pediatrics, Samsung Medical Center, Sungkyunkwan University School of Medicine, Seoul, Korea
}

GLB1-related disorders comprise two phenotypically unique disorders: GM1 gangliosidosis and Morquio B disease. These autosomal recessive disorders are caused by $\beta$-galactosidase deficiency. A hallmark of GM1 gangliosidosis is central nervous system degeneration where ganglioside synthesis is highest. The accumulation of keratan sulfate is the suspected cause of the bone findings in Morquio B disease. GM1 gangliosidosis is clinically characterized by a neurodegenerative disorder associated with dysostosis multiplex, while Morquio B disease is characterized by severe skeletal manifestations and the preservation of intelligence. Morquio B disease and GM1 gangliosidosis may be on a continuum of skeletal involvement. There is currently no effective treatment for GLB1-related disorders. Recently, multiple interventions have been developed and there are several ongoing clinical trials.

Key words: GM1 gangliosidosis, Mucopolysaccharidoses IVB, Morquio B disease, Beta-galactosidase, GLB1.

\section{Introduction}

GLB1-related disorders comprise two phenotypically unique disorders, GM1 gangliosidosis (MIM 230500) and Morquio B disease (mucopolysaccharidosis type IVB, MPS IVB, MIM 253010). GM1 gangliosidosis and Morquio B disease are autosomal recessive disorders caused by $\beta$-galactosidase deficiency. $\beta$-galactosidase is a lysosomal hydrolase involved in sphingolipid GM1 ganglioside metabolism. It may be defective for gangliosides, lactosylceramide, asialofetuin, oligosaccharides carrying terminal $\beta$-linked galactose, and keratan sulfate in GM1 gangliosidosis, and for keratan sulfate alone in Morquio B disease $[1,2]$. When enzyme activity is decreased, sphingolipid intermediates accumulate in the lysosome and interfere with appropriate organelle functioning. A hallmark of GM1 gangliosidosis is degeneration of the central nervous system (CNS), where ganglioside synthesis is highest. Keratan sulfate accumulation is the suspected causative agent for the bone findings associated with Morquio B disease. This review focused on the clinical, radiographic, and genetic characteristics of these two disorders and introduced recent clinical trials on GLB1-related disorders.

\section{Pathogenesis of GLB1-related Disorders}

Both Morquio B and GM1 gangliosidosis arise from mutations in the $G L B 1$ gene. Depending on the location of the mutations in the GLB1 gene and on their combination in compound heterozygous individuals, the molecular pathophysiology of the resulting $\beta$-galactosidase protein can produce a spectrum of phenotypic presentations, ranging from primarily neurologic manifestations in GM1-gangliosidosis, to primarily skeletal involvement in Morquio B disease [3]. Emerging but limited evi-

Received: 4 May 2021, Revised: 8 June 2021, Accepted: 9 June 2021, Published: 30 June 2021

*Corresponding author: Dong-Kyu Jin, M.D., Ph. D. (iD https://orcid.org/0000-0003-4162-2706

Department of Pediatrics, Samsung Medical Center, Sungkyunkwan University School of Medicine, 81 Irwon-ro, Gangnam-gu, Seoul 06351, Korea.

Tel: +82-2-3410-3525, Fax: +82-2-3410-0043, E-mail: jindk.jin@samsung.com

Conflict of interest: The authors declare that they do not have any conflicts of interest.

(c) This is an open-access article distributed under the terms of the Creative Commons Attribution Non-Commercial License (http://creativecommons.org/licenses/by-nc/4.0/) which permits unrestricted non-commercial use, distribution, and reproduction in any medium, provided the original work is properly cited.

(c) Copyright 2021 by the Korean Society of Medical Genetics and Genomics 
dence suggests cytokines may play a role in the pathophysiology of inflammation and pain in patients with Morqio B disease. According previous study of the in vitro, the intracellular glycosaminoglycan storage can potentially trigger the inflammatory response via apoptosis of connective tissue cells, cartilage breaking up, and subsequent elevation of pro-inflammatory cytokines [4]. The articular cartilage also is thicker in Morquio animals with a hyperplastic synovium, suggesting that there are also abnormalities in articular cartilage matrix formation [4].

The clinical manifestations of GM1 gangliosidosis result from the massive storage of GM1 ganglioside and related glycoconjugates in different tissues and particularly in the CNS. However, the molecular mechanisms leading to the disease pathogenesis are still incompletely understood. Neuronal cell death and demyelination accompanied by astrogliosis and microgliosis are usually observed in areas of severe neuronal vacuolation. Neuronal apoptosis, endoplasmic reticulum stress response $[5,6]$, abnormal axoplasmic transport resulting in myelin deficiency [6], and disturbed neuronal-oligodendroglial interactions [7] have been proposed as possible pathomechanisms involved in GM1 gangliosidosis.

\section{Clinical and Radiographic Characteristics of GM1 Gangliosidosis}

The incidence of GM1 gangliosidosis is approximately $1 / 100,000-200,000$ live birth in the general population [8]. This is a progressive neurodegenerative disorder whose manifestations are caused by an accumulation of gangliosides in the CNS and glycosaminoglycans and glycopeptides in the visceral and skeletal tissues [9]. GM1 gangliosidosis is classified into the infantile form (type I), late-infantile form (type Ila)/juvenile form (type IIb), and adult form (type III), according to the age of symptom onset and severity.

The infantile form is the most common and usually presents with poor sucking and inadequate weight gain during the neonatal period. This is characterized by psychomotor regression by the age of six months, visceromegaly, a cherry-red spot, and facial and skeletal abnormalities [1]. Cardiomyopathy and seizures are common, and some infants manifest hepatosplenomegaly. Most infants are blind and deaf with severe CNS dysfunction leading to decerebrate rigidity by the end of their first year [10]. Disease progression is rapid, with death by age two to three years due to respiratory complication. The late-infantile/ and juvenile forms have heterogeneous findings. Patients with the late-infantile form usually present between one and three years of age and have a life expectancy of five to ten years. These patients develop normally until the age of one and then demonstrate developmental regression at 12 to 18 months of age. They have corneal clouding, neurologic abnormalities such as motor abnormalities, and progressive diffuse brain atrophy on brain imaging. The juvenile form presents between 3 to 10 years old. Major symptoms include motor and/or language regression. These children develop normally and suddenly manifest neurologic abnormalities such as progressive dysarthria. The speed of juvenile form's progression is slower than that of the lateinfantile form. Plateauing of motor and cognitive development is followed by slow skill regression. The juvenile form may or may not involve skeletal dysplasia. Life expectancy for this form is well into the second decade [11]. The adult form is a slowly progressive disease involving spasticity, ataxia, dysarthria, and a gradual loss of cognitive function $[9,11]$. These symptoms are similar to the extrapyramidal signs in Parkinson's disease [12], found in 95\% of individuals. The adult form shows short stature, kyphosis, and scoliosis of varying severity. Prognosis depends on the degree of neurologic impairment [10].

The infantile form routinely has severe skeletal dysplasia identified after neurological symptoms. Intervention for these skeletal findings is often limited due to the short life span of these patients [10]. The adult form has few skeletal findings, usually not requiring surgical interventions. One study described skeletal phenotype in patients with GM1 gangliosidosis [13] and described radiographic skeletal abnormalities in 13 late infantile and 21 juvenile patients. The prevalence of odontoid hypoplasia and pear-shaped vertebral bodies was statistically higher in patients with the late-infantile form GM1 gangliosidosis, while vertebral body endplate irregularities and central indentations and squared vertebral bodies were statistically more common in patients with the juvenile form GM1 gangliosidosis. Those with the juvenile form showed less severe pelvic and femoral anomalies than those with the late-infantile form. On the other hand, all patients with GM1 gangliosidosis demonstrated no carpal bone, metacarpal, or phalange abnomality. Notably, and in contrast to the MPS, the short tubular bones are not affected in either late infantile or juvenile GM1 gangliosidosis patients, which distinguishes them from other causes of dysostosis multiplex. Brain magnetic resonance imagings (MRIs) can show diffuse atrophy and white matter abnormalities, non-specific T2-weighted hypointensity in the basal ganglia/globus pallidus, hyperintensity in the putamen, and/or mild cerebral atrophy [11]. A 26-year-old Korean woman with the late-infantile form of GM1 gangliosidosis showed an increased signal intensity of the white matter, 
thalami, and peripheral putamen, and diffuse cerebral and cerebellar hemisphere atrophy in the brain MRI (Fig. 1).

\section{Diagnosis of GM1 Gangliosidosis}

Formal diagnostic criteria have not been established for GLB1related disorders [11]. Most patients with GM1 gangliosidosis are diagnosed through clinical suspicion and subsequent enzyme assays and genetic testing. GM1 gangliosidosis diagnosis is challenging when only based on clinical information and imaging results. It is also difficult to discriminate GM1 gangliosidosis from other neurodegenerative disorders, specifically for the lateinfantile/juvenile form $[14,15]$. Poor disease awareness and nonspecific clinical symptoms complicate and delay diagnosing GM1 gangliosidosis [9].

Recently, a study reported eight Korean patients with lateinfantile GM1 gangliosidosis who were diagnosed by wholeexome sequencing (WES). This paper noted hypomyelination on the brain MRI, elevated aspartate transaminase levels, and skeletal survey abnormalities as important clues of a GM 1 gangliosidosis diagnosis [15]. These patients demonstrated a non-classical presentation [15]. Although WES is not a first tier diagnostic option, genetic tests such as WES can aid in the early diagnosis of rare disorders like GM1 gangliosidosis with a nonclassical presentation.

According to Human Gene Mutation Database (version 2021.01), 252 disease-causing mutations have been identified in GLB1. The most common mutations are missense/nonsense (74.2\%), followed by small deletion (9.1\%) and splicing substitutions (7.9\%). GLB1 mutation's hotspot region appears to cluster in exons 2, 6, and 15 [16]. The recurrent mutation, p.D448V, likely causes the late-infantile form of GM1 gangliosidosis in Korean individuals, although it was previously reported to be a causative mutation of the infantile form of gangliosidosis in patients from
China and Turkey [15]. The genotype-phenotype correlation is not definite [17].

\section{Clinical and Radiographic Characteristics of Morquio B Disease}

Morquio B disease is characterized by severe skeletal manifestations (dysostosis multiplex), growth retardation, corneal clouding, and cardiac valvular disease. Primary CNS involvement has not been proven [1]. The prevalence of Morquio B disease is much lower than that of $\mathrm{GM} 1$ gangliosidosis. The estimated incidence of Morquio B disease is 1:250,000-1,000,000 live births [18]. The severe form of Morquio disease usually becomes apparent between the ages of one and three years [19], and kyphoscoliosis, genu valgum, and pectus carinatum are the most common initial manifestations. The attenuated form of Morquio disease may not be evident until late childhood or adolescence, and hip problems, including pain, stiffness, and Legg-Perthes disease, are common initial manifestations [20,21]. Extraskeletal problems can lead to significant morbidity, including respiratory compromise, obstructive sleep apnea, valvular heart disease, hearing impairment, corneal clouding, dental abnormalities, and hepatomegaly. Specifically, spinal cord compression can result in neurologic compromise in people with severe disease or delayed diagnosis [22]. Coarse facial features can develop later in life, but these changes are milder than those observed in other MPS. Children with Morquio disease typically have normal intellectual abilities. Ligamentous laxity and joint hypermobility, rare in other MPS, are distinctive features of Morquio disease. Short stature with a disproportionally short trunk is a constant feature in Morquio B disease. Younger children are still within the normal height range, but adults are significantly below the third percentile [3]. The structural and degenerative bone, cartilage, and connective tissue changes decrease quality of life due

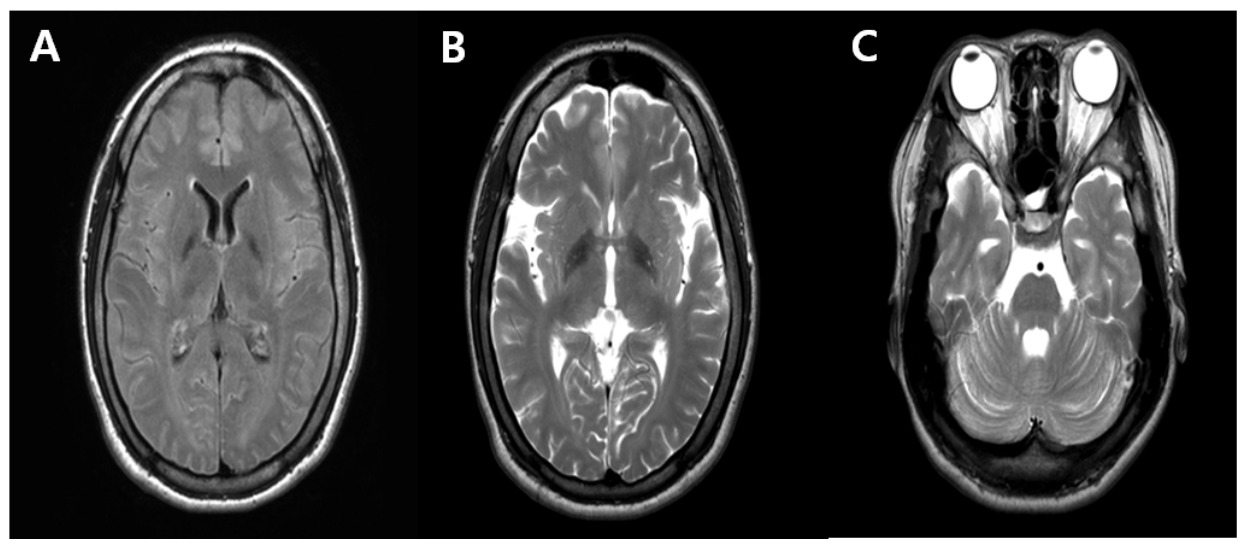

Fig. 1. Brain MRI of a woman with lateinfantile form GM1 gangliosidosis, age 26. (A) T2 FLAIR axial image showing mildly increased signal intensity of the white matter, thalami, and peripheral putamen. $(B, C)$ T2 axial images showing diffuse atrophy of the cerebral and cerebellar hemispheres. 
to mobility issues and limitations in self-care activities. In one Morquio B disease patient/caregiver survey [23], the majority of respondents were using walking aids by adolescence. Moreover, respondents reported an average of three orthopedic surgeries per person, with hip and knee replacement most frequently performed in the second decade of life.

A skeletal survey should be done for MPS IVB diagnosis. Radiographic findings include odontoid hypoplasia, spinal canal narrowing, vertebral beaking, platyspondyly, hip dysplasia, carpal and tarsal dysplasia, and shortening and epi-and metaphyseal dysplasia of the long bones [3]. A 14-year-old Korean boy with Morquio B disease showed J-shaped sella, short and thick metacarpal and phalangeal bones, paddle-shaped ribs, poorly formed bilateral acetabular roofs with flared iliac wings, a flattened femoral head, thoracolumbar spine subluxation (T12-L1), and bilateral genu valgum (Fig. 2).

\section{Diagnosis of Morquio B Disease}

Keratan sulfate accumulates in the bones and cartilage in Morquio B disease, in contrast to GM1 gangliosidosis, where the main accumulating substrates are GM1 and GA1 gangliosides in CNS. Keratan sulfate can be measured quantitatively by LC-MS/
MS-based technologies [24]. Increased keratan sulfate in the urine can be diagnostic of Morquio disease; however, a glycosaminoglycan screen can be falsely negative. Testing to confirm the diagnosis should be performed if there is clinical suspicion. Enzyme activities should be measured for differentiation between the two diseases. Despite the availability of molecular genetic testing, the mainstay of GLB1-related disorder diagnosis is enzyme activity due to the cost and difficulty in interpreting variants of unknown significance. Diagnosis of Morquio B disease can be confirmed by the combination of keratan sulfate in the urine and decreased $\beta$-galactosidase enzyme activity in peripheral blood leukocytes or fibroblasts in the absence of intellectual disability [11].

Among 252 disease-causing mutations identified in GLB1, missense are most common (86.3\%). There has been reported only one patient with Morquio B disease in Korea, and he had mutations c.367G>A (p.G123R), c.13_14insA (p.L5Hfs*29) in GLB1 [25]. c.13_14insA (p.L5Hfs*29) was novel mutation. The genotype-phenotype correlation is not definite.

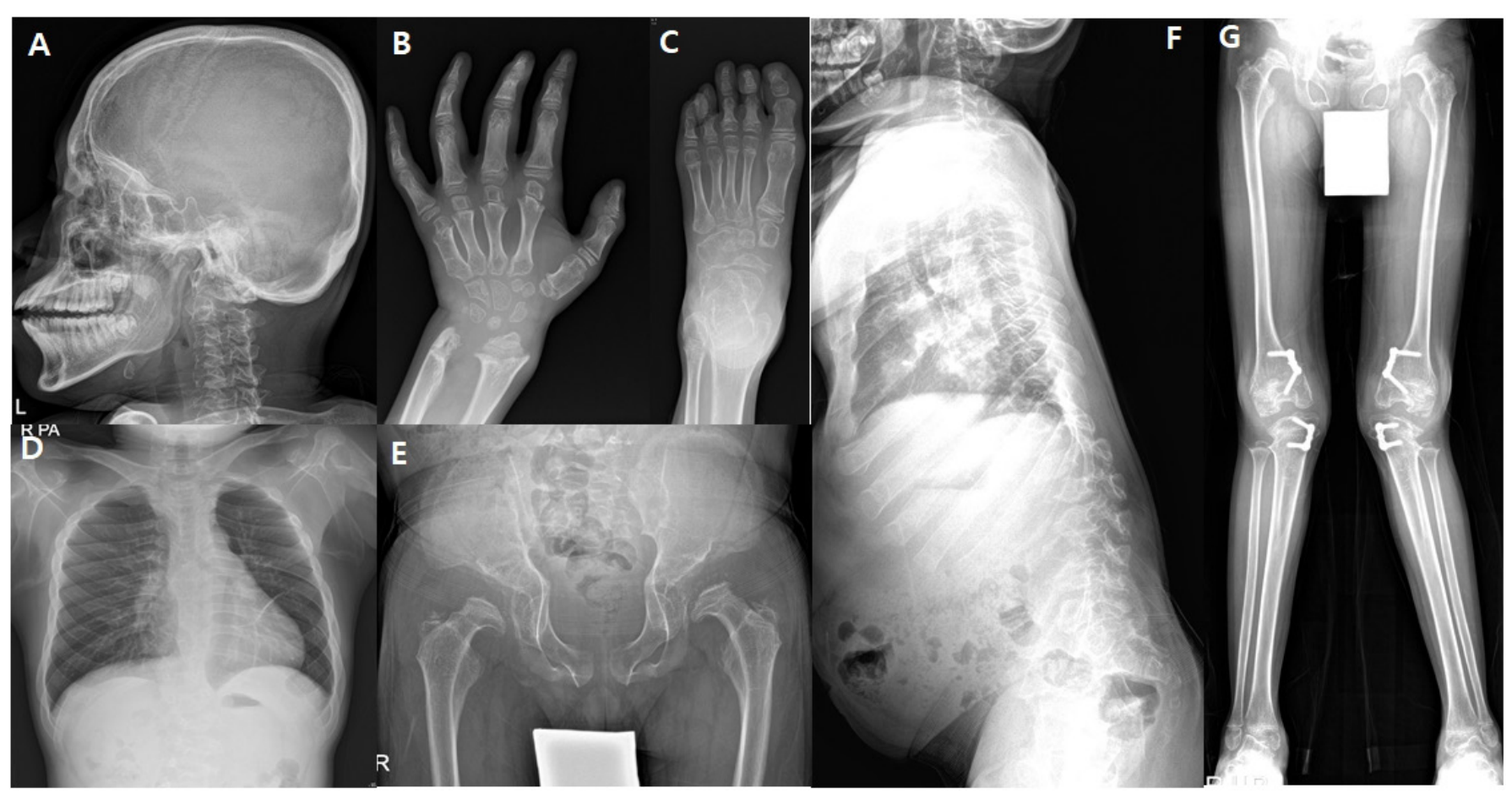

Fig. 2. Skeletal survey of a boy with Morquio B disease, age 14. (A) J- shaped sella. (B) The medial tilted appearance of the end of the radius and ulna and short and thick metacarpal and phalangeal bones. (C) Short and thick metatarsal bones. (D) Short and thick clavicles and paddle-shaped ribs. (E) The flattened femoral head and poorly formed bilateral acetabular roofs with flared iliac wings. (F) Beaking spine with T12-L1 subluxation. (G) Bilateral genu valgum and anteromedial hemiepiphysiodesis of both knees. 


\section{Overlap between Morquio B Disease, MPS IVA, and GM1 Gangliosidosis}

MPS IVA accounts for more than 95\% of affected individuals with the Morquio phenotype, and Morquio B disease accounts for fewer than $5 \%$ of affected individuals. The prevalence range of MPS IVA was estimated from 1 in 76,000 to 1 in 640,000 births $[26,27]$. While Morquio $B$ disease is genetically an allelic variant of GM1 gangliosidosis, it clinically is a mild phenocopy of GALNS-related MPS IVA. Dysostosis multiplex is a common clinical presentation in both Morquio B disease and MPS IVA. While the radiographic findings in MPS IVA and Morquio $B$ disease are extensive and can be diagnostic, they cannot distinguish MPS IVA from MPS IVB. Both diseases show a peculiar type of spondylo-epiphyseal dysplasia with or without additional neuronopathic manifestations. Despite these similarities, the degree of dysostosis multiplex is milder in Morquio B disease compared to MPS IVA [28-30]. The presence of keratan sulfate in the urine does not distinguish MPS IVA from Morquio B disease. Enzyme activities should be measured for differentiation between the two diseases.

GM1 gangliosidosis is clinically characterized by neurodegenerative disorder associated with dysostosis multiplex, while Morquio B disease is characterized by severe skeletal manifestations and the preservation of intelligence. However, an intermediate phenotype between GM1 gangliosidosis and Morquio $B$ disease has recently been proposed in a patient with homozygous p.R333H mutation [31]. Therefore, further neurological follow-up is needed for those patients initially diagnosed with Morquio B disease, since some patients with GM1 gangliosidosis develop neurological impairments later in life [32].

Recently, Ou et al. [17] suggested a three-dimensional analysis, and the in silico outputs of the mutated GLB1 proteins silico tool has helped predict disease severity. However, a clearcut phenotype classification between GM1 types I, II, III, and Morquio $B$ disease is difficult since the vast majority of affected individuals are compound heterozygotes. GLB1 mutations may not be the single determining factor of disease phenotype, as other genes may have an influence [25]. Polymorphisms could also play an interesting role in the resulting enzyme activities and/or phenotypes. It has recently been suggested that patients with a combination of Morquio-like dysostosis and neuronopathic features should be classified as having Morquio B disease plus, while patients with isolated occurrences of Morquio-like dysostosis are classified as having pure Morquio B disease [3].

\section{Preclinical and Clinical Trials of GLB1-related Disorders}

There is currently no effective treatment for GM1 gangliosidosis. There are multiple promising interventions based on in vitro studies and animal models, and a few clinical trials are ongoing. Currently, supportive and symptomatic therapy is the only available treatment option for Morquio B disease, with orthopedic surgeries being the therapeutic mainstay [23].

\section{Pharmacological chaperone therapy}

Pharmacological chaperone therapy with small molecules has advantages in terms of oral administration and broad tissue distribution. Potential compounds have been developed to treat GM1-gangliosidosis and Morquio B disease [33]. Large molecular-weight enzymes cannot cross the blood-brain barrier, so small molecules as possible chaperones for partially functioning $\beta$-galactosidase in the CNS have been investigated. In an in vitro study using fibroblasts from an affected individual, N-octyl4-epi-B-valienamine stabilized $\beta$-galactosidase, reduced lipid accumulation, and improved lipid trafficking [34]. Oral administration of this compound in mice with GM1 gangliosidosis led to increased enzyme activity and reduced substrate levels. Other imino sugar derivatives have shown increased enzyme activity, facilitated localization of $\beta$-galactosidase to lysosomes in the fibroblasts of affected individuals [35], and improved enzyme activity in a mouse model of GM1 gangliosidosis [36].

(5aR)-5a-C-pentyl-4-epi-isofagomine 1, a powerful inhibitor of lysosomal $\beta$-galactosidase and a remarkable chaperone for mutations associated with GM1-gangliosidosis and Morquio disease type $B$, induced mutated $\beta$-galactosidase maturation in fibroblasts of a GM1-gangliosidosis patient. It also promoted the decrease of keratan sulfate and oligosaccharide load in patient cells. This compound is a potential treatment for GM1gangliosidosis and Morquio disease type B patients harboring $\beta$-galactosidase mutations sensitive to pharmacological chaperoning [37].

Recently, in one study involving fibroblasts of a patient with infantile GM1-gangliosidosis, butyl deoxygalactonojirimycin (NB-DGJ) promoted p.D151Y $\beta$-Gal maturation and enhanced its activity up to $4.5 \%$ of control activity within $24 \mathrm{~h}$ and up to $10 \%$ within six days. The NB-DGJ enhancement effect was sustained over three days after it was washed out from culture media. This may be a promising therapeutic chemical chaperone in infantile GM1 amenable variants [38]. 


\section{Substrate reduction therapy}

Miglustat, the imino sugar $\mathrm{N}$-butyl deoxynojirimycin, also showed promising outcomes in a murine model of GM1 gangliosidosis [39]. Miglustat helped slow down or reverse disease progression in juvenile/adult GM1-gangliosidosis [40]. A combination therapy using miglustat and a ketogenic diet is currently in phase 2 [41]. Miglustat administration in four children (20125 months) from Italy affected by the GM1 gangliosidosis type I was safe and relatively well tolerated. Three children had stabilized and/or slowed down neurological progression [42].

\section{Gene therapy}

Vascular delivery of an AAV9 vector encoding $\beta$ gal showed successful widespread expression of functional enzyme throughout the CNS, reducing GM1-ganglioside storage and significantly extending lifespan with retention of motor function in adult GM1 mice [43]. A phase 1/2 study of intravenous gene transfer with an AAV9 vector expressing human galactosidase in type II GM1 gangliosidosis is ongoing (NCT03952637). GLB1 knockout cerebral organoids microinjected with the AAV9GLB1 vector showed a significant increase in $\beta$-gal activity and a significant reduction in GM1 ganglioside content compared with AAV9-GFP-injected organoids, demonstrating the efficacy of an AAV9 gene therapy-based approach in GM1 gangliosidosis [44]. A phase 1/2 study of a single dose of PBGM01 (AAVHu68 vector expressing human galactosidase) delivered into the cisterna magna in type 1 and type 2a infantile GM1 gangliosidosis is currently under way (NCT04713475).

\section{RTB lectin}

RTB lectin is the non-toxic carbohydrate-binding subunit B of ricin toxin that has a high affinity for galactose/galactosaminecontaining glycolipids and glycoproteins that are commonly found on human cell surfaces. In vivo biodistribution studies in lysosomal storage disease models provide evidence that RTB-lectin transports corrective doses of enzymes across the blood-brain barrier to treat CNS pathologies [45]. Plant-made $\beta$-gal:RTB was efficiently taken into GM1 patient fibroblasts and mediated the reduction of GM1 ganglioside substrate [46]. Uptake-saturation analyses indicated that the RTB adsorptivemediated mechanisms of $\beta$-gal:RTB supported a significantly greater accumulation of $\beta$-galactose activity in fibroblasts compared to the receptor-mediated mechanisms of the mammalian cell-derived $\beta$-gal.

\section{Intracerebraventricular enzyme injection}

A single intracerebroventricularly (ICV) administered dose of rh $\beta$-gal $(100 \mu \mathrm{g})$ resulted in broad bilateral biodistribution of $\mathrm{rh}$ $\beta$-gal to critical pathological regions in a mouse model of GM1 gangliosidosis. Weekly ICV dosing of rh $\beta$-gal for eight weeks substantially reduced brain levels of ganglioside and oligosaccharide substrates and reversed well-established secondary neuropathology [47].

\section{Intravenous injections of the fusion enzyme}

A novel fusion enzyme, labeled mTfR-GLB1, was developed by fusing $\beta$-gal to the mouse monoclonal antibody against the mouse transferrin receptor and tested in a murine model of GM1-gangliosidosis. This study showed that mTfR-GLB1 was a catalytically active $\beta$-gal fusion enzyme in vivo that is readily taken into tissues. However, behavior tests showed limited or no improvement, and administration of mTfR-GLB1 was insufficient to create measurable increases in $\beta$-gal enzyme activity in the brain or to reduce ganglioside content [48].

\section{Conclusion}

GLB1-related ailments comprise two phenotypically unique disorders. GM1 gangliosidosis is clinically characterized by a neurodegenerative disorder associated with dysostosis multiplex, and Morquio B disease is characterized by severe skeletal manifestations and preservation of intelligence. There have been several recent interventions and ongoing clinical trials. Knowledge of the natural history and genotype-phenotype correlation of GLB1-related conditions is essential to determine future clinical trial outcomes. A multi-center database study for the longitudinal observation of patients with GLB1-related disorders is needed.

\section{Acknowledgements}

We wish to thank all of the individuals who are living with rare diseases, their families, and the clinical and research laboratory staff.

\section{Authors' Contributions}

Conception and design: SYC. Acquisition of data: SYC. Analysis and interpretation of data: SYC. Drafting the article: SYC. Critical revision of the article: DKJ. Final approval of the version to be published: DKJ. 


\section{References}

1. Suzuki Y, Oshima A, Nanba E. $\beta$-Galactosidase deficiency ( $\beta$-galactosidosis): GM1 gangliosidosis and Morquio B disease. In: Scriver CR, Beaudet AL, Valle D, Sly WS, eds. The metabolic and molecular bases of inherited disease. 8th ed. New York: McGraw-Hill, 2001;3775-809.

2. Caciotti A, Donati MA, Bardelli T, d'Azzo A, Massai G, Luciani L, et al. Primary and secondary elastin-binding protein defect leads to impaired elastogenesis in fibroblasts from GM1-gangliosidosis patients. Am J Pathol 2005;167:1689-98.

3. Abumansour IS, Yuskiv N, Paschke E, Stockler-Ipsiroglu S. Morquio-B disease: clinical and genetic characteristics of a distinct GLB1-related dysostosis multiplex. JIMD Rep 2019;51:30-44.

4. Simonaro $C M$, D'Angelo $M$, Haskins $M E$, Schuchman EH. Joint and bone disease in mucopolysaccharidoses VI and VII: identification of new therapeutic targets and biomarkers using animal models. Pediatr Res 2005:57(5 Pt 1):701-7.

5. Tessitore A, del P Martin $M$, Sano R, Ma $Y$, Mann L, Ingrassia A, et al. GM1-ganglioside-mediated activation of the unfolded protein response causes neuronal death in a neurodegenerative gangliosidosis. Mol Cell 2004;15:753-66.

6. d'Azzo A, Tessitore A, Sano R. Gangliosides as apoptotic signals in ER stress response. Cell Death Differ 2006;13:404-14.

7. Folkerth RD. Abnormalities of developing white matter in lysosomal storage diseases. J Neuropathol Exp Neurol 1999;58:887-902.

8. van der Voorn JP, Kamphorst W, van der Knaap MS, Powers JM. The leukoencephalopathy of infantile GM1 gangliosidosis: oligodendrocytic loss and axonal dysfunction. Acta Neuropathol 2004;107:53945.

9. Brunetti-Pierri N, Scaglia F. GM1 gangliosidosis: review of clinical, molecular, and therapeutic aspects. Mol Genet Metab 2008;94:391-6.

10. Suzuki Y, Nanba E, Matsuda J, Higaki K, Oshima A. $\beta$-Galactosidase deficiency ( $\beta$-Galactosidosis): $G_{M 1}$ gangliosidosis and Morquio $B$ disease. In: Valle DL, Beaudet AL, Vogelstein, B, Kinzler KW, Antonarakis

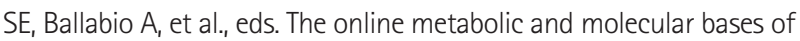
inherited disease. New York: McGraw-Hill, 2014.

11. Regier DS, Tifft CJ, Rothermel CE. GLB1-related disorders. In: Adam MP, Ardinger HH, Pagon RA, Wallace SE, eds. GeneReviews ${ }^{\circledR}$. Seattle: University of Washington, 1993.

12. Roze E, Paschke E, Lopez N, Eck T, Yoshida K, Maurel-Ollivier A, et al. Dystonia and Parkinsonism in GM1 type 3 gangliosidosis. Mov Disord 2005;20:1366-9.

13. Ferreira CR, Regier DS, Yoon R, Pan KS, Johnston JM, Yang $S$, et al. The skeletal phenotype of intermediate GM1 gangliosidosis: clinical, radiographic and densitometric features, and implications for clinical monitoring and intervention. Bone 2020;131:115142.
14. Baiotto $C$, Sperb F, Matte $U$, da Silva $C D$, Sano $R$, Coelho JC, et al. Population analysis of the GLB1 gene in South Brazil. Genet Mol Biol 2011;34:45-8.

15. Lee JS, Choi JM, Lee M, Kim SY, Lee S, Lim BC, et al. Diagnostic challenge for the rare lysosomal storage disease: late infantile GM1 gangliosidosis. Brain Dev 2018;40:383-90.

16. Yang CF, Wu JY, Tsai FJ. Three novel beta-galactosidase gene mutations in Han Chinese patients with GM1 gangliosidosis are correlated with disease severity. J Biomed Sci 2010;17:79.

17. Ou L, Kim S, Whitley CB, Jarnes-Utz JR. Genotype-phenotype correlation of gangliosidosis mutations using in silico tools and homology modeling. Mol Genet Metab Rep 2019;20:100495.

18. Baehner F, Schmiedeskamp C, Krummenauer F, Miebach E, Bajbouj

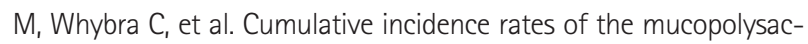
charidoses in Germany. J Inherit Metab Dis 2005;28:1011-7.

19. Montaño AM, Tomatsu S, Gottesman GS, Smith M, Orii T. International Morquio A Registry: clinical manifestation and natural course of Morquio A disease. J Inherit Metab Dis 2007;30:165-74.

20. Hecht JT, Scott Cl Jr, Smith TK, Williams JC. Mild manifestations of the Morquio syndrome. Am J Med Genet 1984;18:369-71.

21. Wraith JE. The mucopolysaccharidoses: a clinical review and guide to management. Arch Dis Child 1995;72:263-7.

22. Tomatsu $S$, Montaño AM, Oikawa $H$, Smith M, Barrera $L$, Chinen $Y$, et al. Mucopolysaccharidosis type IVA (Morquio A disease): clinical review and current treatment. Curr Pharm Biotechnol 2011;12:931-45.

23. Bleier M, Yuskiv N, Priest T, Moisa Popurs MA, Stockler-Ipsiroglu S; BC Children's Hospital; University of British Columbia. Morquio B patient/caregiver survey: first insight into the natural course of a rare GLB1 related condition. Mol Genet Metab Rep 2018;16:57-63.

24. Khan SA, Mason RW, Giugliani R, Orii K, Fukao T, Suzuki Y, et al. Glycosaminoglycans analysis in blood and urine of patients with mucopolysaccharidosis. Mol Genet Metab 2018;125:44-52.

25. Sohn YB, Park HD, Park SW, Kim SH, Cho SY, Ko AR, et al. A Korean patient with Morquio B disease with a novel c.13_14insA mutation in the GLB1 gene. Ann Clin Lab Sci 2012;42:89-93.

26. Khan SA, Peracha $H$, Ballhausen $D$, Wiesbauer A, Rohrbach M, Gautschi $M$, et al. Epidemiology of mucopolysaccharidoses. Mol Genet Metab 2017;121:227-40.

27. Nelson J, Crowhurst J, Carey B, Greed L. Incidence of the mucopolysaccharidoses in Western Australia. Am J Med Genet A 2003;123A:310-3.

28. Stockler-Ipsiroglu S, Yazdanpanah N, Yazdanpanah M, Popurs MM, Yuskiv N, Schmitz Ferreira Santos ML, et al. Morquio-like dysostosis multiplex presenting with neuronopathic features is a distinct GLB1related phenotype. JIMD Rep 2021, in press.

29. Beck M, Petersen EM, Spranger J, Beighton P. Morquio's disease 
type B (beta-galactosidase deficiency) in three siblings. S Afr Med J 1987;72:704-7.

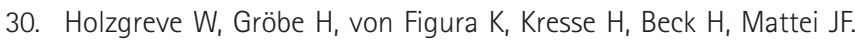
Morquio syndrome: clinical findings in 11 patients with MPS IVA and 2 patients with MPS IVB. Hum Genet 1981;57:360-5.

31. Mayer FQ, Pereira Fdos S, Fensom AH, Slade C, Matte U, Giugliani R. New GLB1 mutation in siblings with Morquio type B disease presenting with mental regression. Mol Genet Metab 2009;96:148.

32. Paschke $E_{1}$ Milos I, Kreimer-Erlacher H, Hoefler G, Beck M, Hoeltzenbein $M_{1}$ et al. Mutation analyses in 17 patients with deficiency in acid beta-galactosidase: three novel point mutations and high correlation of mutation W273L with Morquio disease type B. Hum Genet 2001;109:159-66.

33. Front $S$, Biela-Banaś $A$, Burda $P$, Ballhausen $D$, Higaki $K$, Caciotti $A$, et al. (5aR)-5a-C-Pentyl-4-epi-isofagomine: a powerful inhibitor of lysosomal $\beta$-galactosidase and a remarkable chaperone for mutations associated with GM1-gangliosidosis and Morquio disease type B. Eur J Med Chem 2017;126:160-70.

34. Higaki K, Li L, Bahrudin U, Okuzawa S, Takamuram A, Yamamoto K, et al. Chemical chaperone therapy: chaperone effect on mutant enzyme and cellular pathophysiology in $\beta$-galactosidase deficiency. Hum Mutat 2011;32:843-52.

35. Fantur KM, Wrodnigg TM, Stütz AE, Pabst BM, Paschke E. Fluorous iminoalditols act as effective pharmacological chaperones against gene products from $\mathrm{GLB}_{1}$ alleles causing GM1-gangliosidosis and Morquio B disease. J Inherit Metab Dis 2012;35:495-503.

36. Takai $T$, Higaki $K$, Aguilar-Moncayo M, Mena-Barragán $T$, Hirano $Y_{1}$ Yura $K_{1}$ et al. A bicyclic 1-deoxygalactonojirimycin derivative as a novel pharmacological chaperone for GM1 gangliosidosis. Mol Ther 2013;21:526-32.

37. Front $\mathrm{S}$, Almeida S, Zoete V, Charollais-Thoenig J, Gallienne E, Marmy $C_{1}$ et al. 4-epi-Isofagomine derivatives as pharmacological chaperones for the treatment of lysosomal diseases linked to $\beta$-galactosidase mutations: improved synthesis and biological investigations. Bioorg Med Chem 2018;26:5462-9.

38. Mohamed FE, Al Sorkhy M, Ghattas MA, Al-Gazali L, Al-Dirbashi O, Al-Jasmi $F_{1}$ et al. The pharmacological chaperone N-n-butyl-deoxygalactonojirimycin enhances $\beta$-galactosidase processing and activity in fibroblasts of a patient with infantile GM1-gangliosidosis. Hum Genet 2020;139:657-73.
39. Elliot-Smith E, Speak AO, Lloyd-Evans E, Smith DA, van der Spoel $A C$, Jeyakumar $M$, et al. Beneficial effects of substrate reduction therapy in a mouse model of GM1 gangliosidosis. Mol Genet Metab 2008:94:204-11.

40. Deodato F, Procopio E, Rampazzo A, Taurisano R, Donati MA, Dionisi-

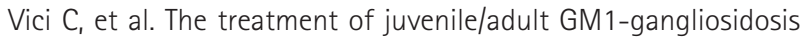
with Miglustat may reverse disease progression. Metab Brain Dis 2017;32:1529-36.

41. Jarnes Utz JR, Kim S, King K, Ziegler R, Schema L, Redtree ES, et al. Infantile gangliosidoses: mapping a timeline of clinical changes. Mol Genet Metab 2017;121:170-9.

42. Fischetto $R$, Palladino $V$, Mancardi MM, Giacomini T, Palladino $S$, Gaeta $A_{1}$ et al. Substrate reduction therapy with Miglustat in pediatric patients with GM1 type 2 gangliosidosis delays neurological involvement: a multicenter experience. Mol Genet Genomic Med 2020;8:e1371.

43. Weismann CM, Ferreira J, Keeler AM, Su Q, Qui L, Shaffer SA, et al. Systemic AAV9 gene transfer in adult GM1 gangliosidosis mice reduces lysosomal storage in CNS and extends lifespan. Hum Mol Genet 2015;24:4353-64.

44. Latour $Y$ L, Yoon R, Thomas SE, Grant C, Li C, Sena-Esteves M, et al. Human GLB1 knockout cerebral organoids: a model system for testing AAV9-mediated GLB1 gene therapy for reducing GM1 ganglioside storage in GM1 gangliosidosis. Mol Genet Metab Rep 2019;21:100513.

45. Acosta W, Cramer CL. Targeting macromolecules to CNS and other hard-to-treat organs using lectin-mediated delivery. Int J Mol Sci 2020;21:971.

46. Condori J, Acosta W, Ayala J, Katta V, Flory A, Martin R, et al. Enzyme replacement for GM1-gangliosidosis: uptake, lysosomal activation, and cellular disease correction using a novel $\beta$-galactosidase:RTB lectin fusion. Mol Genet Metab 2016;117:199-209.

47. Chen JC, Luu AR, Wise N, Angelis R, Agrawal V, Mangini L, et al. Intracerebroventricular enzyme replacement therapy with $\beta$-galactosidase reverses brain pathologies due to GM1 gangliosidosis in mice. J Biol Chem 2020;295:13532-55.

48. Przybilla MJ, Stewart C, Carlson TW, Ou L, Koniar BL, Sidhu R, et al. Examination of a blood-brain barrier targeting $\beta$-galactosidasemonoclonal antibody fusion protein in a murine model of GM1gangliosidosis. Mol Genet Metab Rep 2021;27:100748. 